\title{
A SIMPLIFIED MODELING APPROACH FOR HUMAN SYSTEM INTERACTION
}

\author{
Torbjörn P.E. Ilar \\ Div. of Manufacturing Systems Engineering \\ Luleå University of Technology \\ 97187 Luleå, Sweden
}

\begin{abstract}
Despite increasing dependency on technology, the importance of humans is expected to increase and to provide a realistic basis for decision support; both technical and organizational processes must be included in simulation models. In a rapidly changing environment, skill development is also important and should be considered when developing simulation models. This paper describes a three step straightforward method that support the implementation of supporting (human dependent) processes, operators competence and competence development using learning curves to obtain a more representative simulation of system performance. The effect on the performance measures are also demonstrated based on a case study of a highly automated press line. The advantages and disadvantages with the proposed method are also discussed.
\end{abstract}

\section{INTRODUCTION}

Modern production simulation software makes it quite easy to build models that consider many system variables and which reproduce the dynamic interactions of resources and the time dependence of activities. However, despite the sophistication of the technique, it has been observed that gaps can exist between the performance of the system predicted by DES models and the output which the real system generates in practice.

The situation described by above has been found to occur to a wider extent when the systems contain more human elements (Baines and Kay 2002). Modeling practitioners tend traditionally to consider people quite simplistically and to concentrate on the prediction, planning and positioning of the technological elements of the systems.

One approach to modeling skill and competence development within an industrial organization is the use of a theoretical learning curve. Learning curve theory has been applied to a wide range of industrial applications that include human operators in complex tasks such as assembly (Towill and Cherrington 1994).

Rita Freudenberg and Henry Herper (Freudenberg and Herper 1998) describe an approach to the simulation of workers in manufacturing systems. They stress the importance of modeling workers in varying levels of detail which match the level of detail in the simulation model.

A more accurate representation of worker's behavior could be provided within the DES technique through a human performance modeling tool that integrates with computer-based simulation to give a holistic view of system performance (Baines and Kay 2002; Baines et al 2005). The human performance modeling tool is passing information about worker performance to a DES model and taking information on the factors that influence this performance as an input. To create such a modeling tool requires an in-depth understanding of the determinants of worker behavior. In other words, a framework is needed to capture the worker's key rules, assumptions, and relationships.

The human performance model suggested by Baines and Benedettini (Baines and Benedettini 2007) is very complex and includes individual aspects (i.e. extroversion, job satisfaction, age and work attitude), the physical environment (i.e. noise level, temperature and ventilation) and the organizational environment (i.e. shift patterns, work teams, training, job rotation and hierarchical structure). This is a very ambitious approach but is also very challenging for simulation modelers.

\section{A HUMAN MODELING METHOD}

The research presented in the previous section is very ambitious but their approach might, in many cases, be too complex to be industrially applicable. The positive relationship between the efforts required and advantages of this approach might be questionable. It that sense the proposed more straightforward approach outlined in this paper has greater applicability and can, from the production manager's perspective, include most of the advantages with much less effort. 
The proposed human modeling methods consist of three sequential and interacting steps outlined in Figure 1 below.

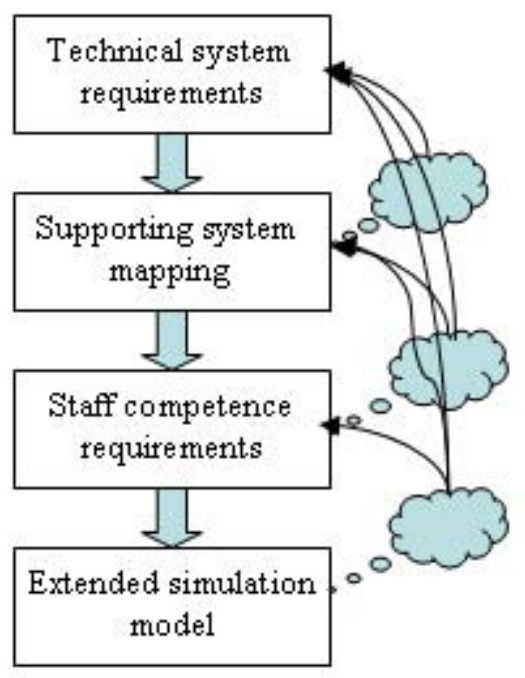

Figure 1: Human modeling method

The technical systems requirements is of course an important step and will follow the standard procedure applied within industry and will not be further addressed in this paper. However it is of great importance that the development of the technical system requirements involves informal expertise in form of shop floor operators (the process experts).

The development of the supporting systems maps and staff competence requirements can lead to suggestions for improvement in the design of the previous step (i.e. lack of competence within the team might lead to a redesign of one of the processes within the supporting processes and/or redesign of the technical system to eliminate the specific supporting process.

The contents and purpose of the three last steps in the method is described in section $2.1-2.3$.

\subsection{Supporting system mapping}

One important feature of the method is its focus on the integration of the supporting systems into the conceptual model of a semi automated production system. This implies, from the perspective of a project manager, a more holistic view the system design including both the technically dependent main process and organizationally dependent supporting processes. The latter demands an extended project group including staff with knowledge of the supporting processes (i.e. operators and shop floor managers).
The method used in the first step is development of process maps, with tools such as $\mathrm{IDEF}_{0}$ (NIST 1993), based on feedback from the informal experts. The approach supports the step by step reduction of system complexity, from process to individual activities, and thus gives operators and technical staff the opportunity to recognize their role in the supporting processes. It thereby provides realistic inputs in terms of activity scope, time and required resources.

\subsection{Staff competence requirements}

The lowest level of the supporting process maps, the activity level, will provides the resources needed in terms of equipment, staffing, task etc. Each task will than requires a certain skill and the purpose with the competence matrix is to identify the team members ability or competence to perform the different tasks required.

This might be seen as a simple step but it is probably the most delicate step in the proposed method. This kind of grading of operators is often sensitive but competence matrix has started to be quite commonly used tools in shop floor and team oriented human management. The way to perform this must be adapted to the culture within the organization but it can be based on interviews with shop floor managers, team leaders or/and operators (member of the team). The main purpose is to identify the initial team competence with respect to the requirements. An example of a competence matrix can be seen in Figure 2.

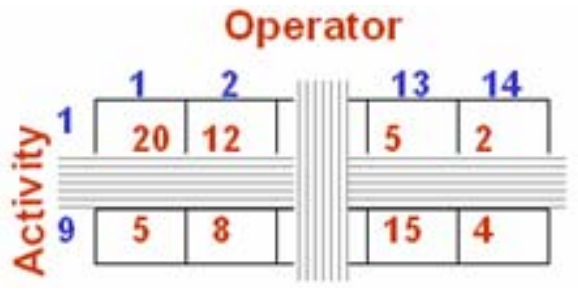

Figure 2: Competence matrix

High number indicates operators with high skill for the specific task.

\subsection{Extended simulation model}

The third step is a production simulation study, based upon data from the process mapping step, to predict and communicate the overall performance of the system taking into account the main and supporting processes and the interaction between these. The use of simulation also provides the project group with performance benchmarks to support the development of the system. An important issue with the model is to scope the interaction between the main and supporting system. 
To be able to model 'learning' associated with the support processes, a competence profile and learning curve is included in the model for two tasks. Two values (which change as the model is run) describes a unique competence profile for each operator.

The competence index for each operator is updated when $\mathrm{s} /$ he performs an activity following a time constant learning model as follows:

$$
\begin{aligned}
& x=x+1 \\
& t u_{i j}=t u_{i j o p t}+\Delta t u_{i t} \times e^{-\frac{X}{C_{t}}}
\end{aligned}
$$

where: $x$ is learning curve index, $t u_{i j}$ is the operation time in the updating matrix for operation $i$ and operator $j$, $\mathrm{tu}_{\mathrm{ijopt}}$ is the optimum operation time, $\Delta t u_{i j}$ is the initial activity time and $c_{t}$ is a learning curve factor.

In summery the extended simulation model has two unique features:

- the detailed modeling of and highly focus on the supporting processes.

- the implementation of team competence development based on learning curve theory.

The main advantages of the extended model are:

- Common understanding of main and supporting processes interactions.

- More realistic performance measures taking technical as well as organizational (supporting processes, competence and competence build up) aspects into account.

- Opportunity to include learning potential in the evaluation of different alternatives.

- More desirable start up situation and thus reduced commissioning time due to:

- actual and active participation from the operators (process owners).

- $\quad$ knowledge capturing.

- parallel and gradual development of the processes.

This is summarized in Figure 3.

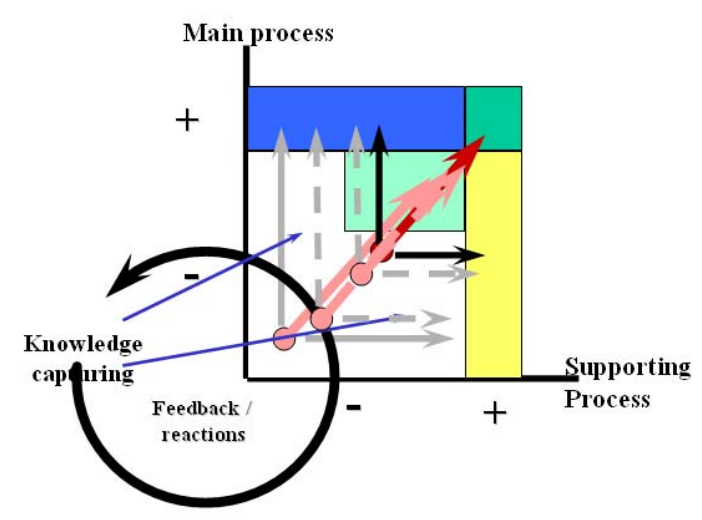

Figure 3: The enhanced model in the development of overall system performance
The overall system improves towards the green square in the upper right corner.

There are also some risks/challengers related to the proposed model such as:

- Requirements of a more cross-functional project team which might be harder to manage.

- The capture of the initial operator competence data.

- Increased modeling time and effort.

- A possible reduction of the model accuracy compared to purely technical models due to less accurate data and more stochastic activities.

- The pedagogical shift from a model with the purpose of providing performance measures to that of providing and communicating insights.

Keeping those advantages and risks in mind I believe that the proposed method can increase model usefulness in the right context. The context must be a system where the performance is expected to be affected by the interaction between the main and supporting processes.

\section{SIMULATION EXPERIMENTS}

\subsection{Project aim and model scope}

The aim of the work presented in this case study was to increase understanding of the impact of worker competence on overall productivity in a recently commissioned highly automated press line. The simulation model developed included both technical (main) and organizational (supporting) processes and focused on the interaction between these.

Whilst production simulation had been used as a decision support tool in the early phases of this project, these initial models covered only the main processes. The effects of operator learning and interaction effects associated with the sub-processes were neglected. These initial models, in retrospect, indicated performance / production levels far higher than seen in practice.

In the present study process mapping was used to identify the processes to be included in the discrete event simulation model. The processes chosen were the main process as well as tool preparation, set-up and maintenance processes all of which had the greatest impact on overall system performance. 


\subsection{Experimental design and model responses}

Factorial design was used when planning the simulation runs. Four factors were of interest which gives a total of 16 combinations to achieve a four factor, two level, full factorial experiment (Law and Kelton 2000). The following factors were included in the simulation experiment:

1. The constant in the learning curve equation. This is mainly for sensitivity analysis since an exact value for this constant is very difficult to find. Level 0 equates to 5 and level 1 to 15 .

2. The level of operator availability. Level 0 represents $90 \%$ and level $170 \%$.

3. Batch size. Level 1 equals 200 and level 0 equals 50.

4. Two different human management philosophies. Level 0 represents an expert concentration philosophy where the operator who will complete the activity in the shortest time is given the highest priority. Level 1 is for a competence spread philosophy where the operator with the longest activity time has the highest priority.

Factor 3 and 4 of this study can be controlled, so that data may be obtained about how these factors influence another variable referred to as the response variable or performance measure.

The experimental study was conducted in two steps. The first step focused on knowledge build up including factors 1 and 4 . In the second step, the best knowledge build up result for each human management philosophy was used as an input and factors 2 and 3 were introduced. The 4 factor composition for the first step and 8 factor compositions for the second step were simulated 5 times to achieve four replicates. This is known as a full experiment run. The mean and the variations could then be calculated for each response. Statistical analysis also generated confidence intervals for each response.

The model responses used were selected with the aim of supporting communication of the behavior of the system under these different scenarios rather then for providing direct measures of the performance of the real system. The following responses were selected:

Relative production rate, which was defined as the actual production rate verses the production rate from the previous decision support simulation run.

Performances of supporting processes, which was the mean time and variations associated with carrying out tool preparation and maintenance processes.

Group competence build up, which including the current competence index divided by the optimum competence (SDO), variation in competence (VIC) and the com- petence build up time. The latter response was defined as when operator group reached a competence index of 50\% of optimum and the first two responses were calculated as:

$$
\begin{aligned}
& S D O=\frac{\sum_{1}^{i} \sum_{1}^{j} t_{u i j}}{\sum_{1}^{i} \sum_{1}^{j} t_{o p t i j}} \quad \text { and } \\
& V I C=\sum_{1}^{i} \sum_{1}^{j}\left(t_{u i j}-\bar{t}\right)^{2}
\end{aligned}
$$

Where: $t u_{i j}$ is the operation time in the updating matrix for operation $i$ and operator $j$, $\mathrm{tu}_{\mathrm{ijopt}}$ is the optimum operation time.

The responses were "measured" after a completed simulation run (50 days).

\section{RESULTS}

Table 1 shows the relative production rate (RPR) in \%, defined as the production rate from this simulation study, vs. the production rate from the previous decision support simulation run that only included the main processes (technical). The results are based on simulation of 10 weeks production preceded by a 10 week competence build up period and with a highest level of workload.

Table 1: Relative production rate [RPR]: mean, min, and max based on $95 \%$ confidence interval.

\begin{tabular}{|c|c|c|c|}
\hline $\begin{array}{c}\text { Management } \\
\text { philosophy }\end{array}$ & Mean & Miri & Max \\
\hline $\begin{array}{c}\text { broad } \\
\text { contjetence }\end{array}$ & 30.18 & 29.43 & 30.93 \\
\hline $\begin{array}{c}\text { dedicated } \\
\text { operator }\end{array}$ & 28.81 & 28.35 & 29.27 \\
\hline
\end{tabular}

The study indicated that the highest initial RPR is achieved using dedicated operators However, developing "broad competence" offers advantages after the build up period and when the workload is increased (Figure 4).

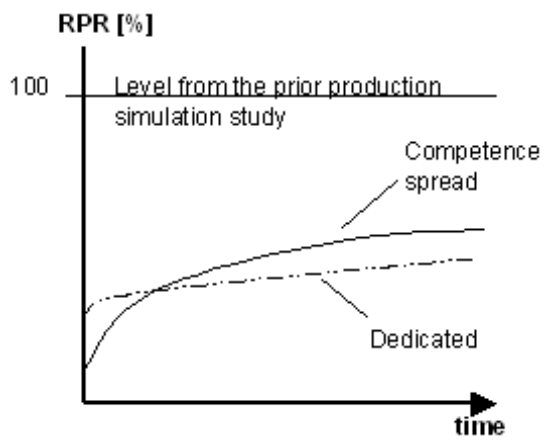

Figure 4: Outline of the RPR development over time for the two human management alternatives. 
Short-term (two shift) measurements of the RPR also shows a much lower variation for the broad competence approach compared to the dedicated operator approach (se Figure 5).

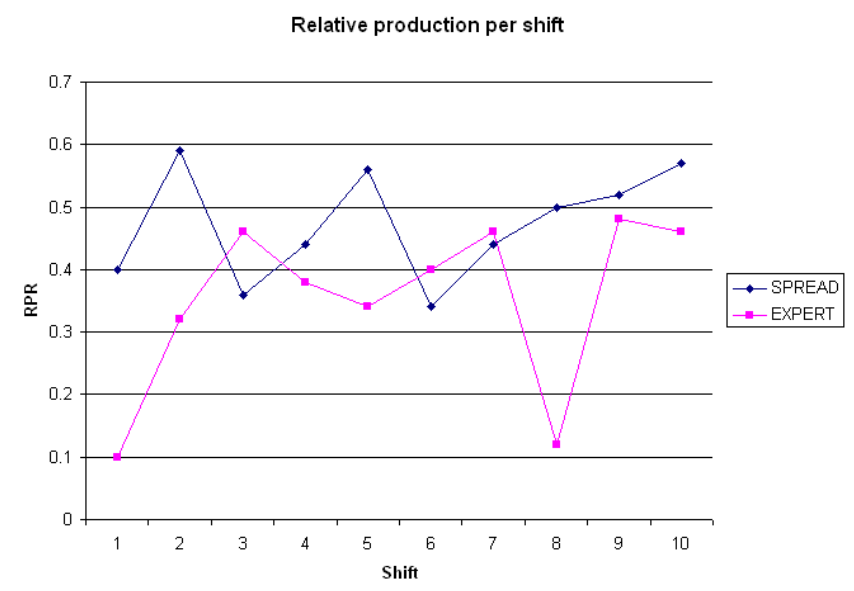

Figure 5: Short term (two shifts) relative production rate [RPR].

Other results of interest are;

- The shortest tool preparation and maintenance process times will be achieved with the expert management approach. However, these times are subject to very high variations due to the inability to handle personnel disturbances.

- The learning curve factor, $c_{t}$, has no significant effect on the 'best' personal management approach. The lower factor value will, of course, lead to a slower group competence build up, but still offer better performance than the competence spread approach.

- A low operator availability combined with small batch sizes is preferable with the management approach of spreading competence. This indicates the group's ability to handle rapid change, which is expected to be of great importance in the next generation of manufacturing systems.

It is important to note that the production rate decreases by approximately $50 \%$ for both alternatives compared to predictions from the earlier study which focused almost entirely on the technical configuration. About one third of this decrease is explained by the more frequent setups in the present study. The remainder is due to the introduction of organizational factors and the inclusion of more realistic breakdown data. This indicates the importance of including both main and supporting process.

\section{CONCLUSIONS}

This paper presents a three steps modeling method to support project management, evaluation and improvement prediction in manufacturing system, where the interaction of the main process with supporting processes is significant. The method uses process mapping and simulation to develop a common understanding of system functionality amongst the project group rather then simply for measuring or predicting system performance.

The case study simulated main and supporting processes as well as using competence profiles to indicate system performance during system start up. The results of the case showed the significant impact that supporting processes can have. Including technical as well as organizational factors is therefore of great importance for understanding the effects of human/machine interaction on the overall system performance.

The most important area of application for the proposed method is to support development of manufacturing systems consisting of highly automated main processes and organizational oriented supporting processes such as were found in the company involved in the case study. Modeling supporting processes drastically improved the quality of the predicted overall system behavior. Using this method will thus significantly increase the usefulness of production simulation to help the project group to understand the interaction between main and supporting processes; something which is often neglected. Furthermore mapping the supporting processes through operator participation in the project work increases involvement and gives valuable feedback in the development of the system.

\section{REFERENCES}

Baines T. S., J. M. Kay. 2002. Human performance modelling as an aid in the process of manufacturing system design: A pilot study, Int. J. for Production Research 40(10):2321-2334

Baines T. S., R. Asch, L. Hadfield, J. P. Mason, S. Fletcher, and J. M. Kay. 2005. Towards a theoretical framework for human performance modelling within manufacturing systems design, Simulation Model Practices and Theory 13(6):486-504

Baines T. S., O. Benedettini. 2007. Modelling of human performance within manufacturing systems design: from theoretical towards practical framework, Journal of Simulation 1(2):121-130

Freudenberg R., H. Herper. 1998, Simulation of workers in manufacturing systems, In Proceedings of the 1998 Winter Simulation Conference, eds. D. J. Medeiros, E. F. Watson, J. S. Carson and M. S. Manivannan, 952956. Piscataway, New Jersey: Institute of Electrical and Electronics Engineers, Inc. 
Law, A. M., and W. D. Kelton. 2000. Simulation modeling \& analysis, 3rd ed. New York: McGraw-Hill, Inc.

NIST. 1993. Integration Definition for Function Modeling (IDEFØ), Draft Federal Information Processing Standards Publication 183, National Institute of Standards and Technology, Gaithersburg, MD, USA.

Towill, D. R., J. E. Cherrington. 1994. Learning Curve Models for Prediction the Performance of AMT. International Journal of Advanced Manufacturing Technology 9(3):195-203

\section{AUTHOR BIOGRAPHY}

TORBJÖRN P.E. ILAR is an Assisted Professor at the division of Manufacturing Systems Engineering at Luleå University of Technology. He has been in the community of production simulation since the mid $80^{\text {th }}$, both as a teacher, researcher and professional consultant. He received a master's degree in industrial management and a $\mathrm{Ph} . \mathrm{D}$. in production simulation from Luleå University of Technology, Sweden. The current research interest is in the context of human machine system interaction and modeling in that area. He is responsible for courses in the field of production management, automation and production simulation. He is also highly active in the development and performance of industrial driven research at the division in the field of modeling of manufacturing systems. He also has an impressive industrial experiences based on accomplishment of about 150 industrial projects and from his small consultants firm which provides expertise and training in production simulation for industries. 